\title{
PERSPECTIVA DA HIPERTENSÃO INTRA-ABDOMINAL E DA SÍNDROME COMPARTIMENTAL ABDOMINAL NO TRANSPLANTE DE FÍGADO: REVISÃO SISTEMATIZADA
}

\author{
Perspective of intra-abdominal hypertension and abdominal compartment syndrome in liver \\ transplantation: A systematic review \\ Luiz Eduardo Rafael Moutinho', Olival Cirilo Lucena da Fonseca Neto²
}

\begin{abstract}
RESUMO
Introdução: No pós-operatório do transplante de fígado pode haver aumento do volume contido no abdômen, reposição maciça de volume, acúmulo de sangue no espaço intraperitoneal e distensão gastrointestinal; essas condições são pertinentes para o desenvolvimento da Hipertensão Intra-Abdominal (HIA) e da Síndrome Compartimental Abdominal (SCA). As evidências acerca desse tema estão em desenvolvimento. O objetivo deste estudo foi fornecer uma visão integrativa da literatura sobre as perspectivas da HIA e SCA no transplante de fígado. Métodos: Foi realizada uma busca sistematizada na MEDLINE (via PubMed), Scielo, LILACS (via BVS) e Cochrane, em 24 de julho de 2019. Os trabalhos foram selecionados conforme critérios de inclusão e exclusão propostos para o objetivo desta revisão e foram divididos em cinco categorias: prevalência, fatores de risco, monitoramento, complicações e manejo. Discussão: A HIA e a SCA são complicações frequentes que podem determinar aumento da morbimortalidade no pós-transplante hepático. Há diversos fatores de risco inerentes ao procedimento para o desenvolvimento dessas condições, devendo, portanto, ser ativamente monitoradas através da PIA medida pelo cateter vesical. A HIA é fator independente para insuficiência renal aguda e pode provocar prejuízo à perfusão hepática. Conclusão: Medidas para o controle e prevenção da HIA e da SCA devem ser tomadas no pós-operatório imediato, incluindo métodos clínicos e cirúrgicos. A manutenção do abdome aberto e a laparotomia descompressiva destacam-se nesse cenário.
\end{abstract}

Descritores: Transplante de fígado; Hipertensão Intra-abdominal; Síndrome do Compartimento Abdominal.

\section{Instituições:}

1- Faculdade de Ciências Médicas, Universidade de Pernambuco Recife/PE, Brasil.

2- Hospital Universitário Oswaldo Cruz. Recife/PE, Brasil.

\section{Correspondência:}

Olival Cirilo Lucena Fonseca Neto.

Unidade de Transplante de Fígado - R.Arnóbio Marquês, 310 - CEP

50100-130, Recife/PE,

Tel.: (81) 99162-6477

E-mail: olivalneto@globo.com

\section{INTRODUÇÃO}

A pressão intra-abdominal (PIA) é a medida da pressão existente na cavidade abdominal. De acordo com a World Society on Abdominal Compartment Syndrome (WSACS), os valores fisiológicos em indivíduos hígidos variam entre 2 e $7 \mathrm{mmHg}$ e a elevação sustentada acima de $12 \mathrm{mmHg}$ leva ao quadro de Hipertensão Intra-Abdominal (HIA). São definidos quatro graus de HIA segundo o perfil de pressão: grau I (12-15 $\mathrm{mmHg})$, grau II (16-20 mmHg), grau III (21-25 $\mathrm{mmHg})$ e grau IV (>25 mmHg). ${ }^{1}$

A instalação de estado hipertensivo dentro do abdome é causada pela diminuição da complacência da parede abdominal, aumento do conteúdo de lesões intra-abdominais, aumento do conteúdo intraluminal 
gastrointestinal e aumento da permeabilidade e/ou do extravasamento capilar. ${ }^{1} \mathrm{O}$ aumento da PIA repercute hemodinamicamente sobre diversos sistemas, sobretudo na perfusão dos órgãos abdominais: a pressão de perfusão abdominal é importante parâmetro nesse contexto, definida pela subtração da pressão arterial média (PAM) pela PIA: PPA=PAM-PIA. ${ }^{2}$

A HIA na UTI tem prevalência importante, variando em torno de $32-51 \%$ nos maiores estudos prospectivos, sendo fator independente de mortalidade. ${ }^{3,4}$ A HIA de modo crítico pode evoluir para síndrome compartimental abdominal (SCA), condição clínica definida pelo aumento sustentado da PIA acima de $20 \mathrm{mmHg}$ associada à nova disfunção orgânica. O conjunto de repercussões da HIA contribui para a disfunção múltipla de órgãos, com ameaça grave à vida do paciente. A laparotomia descompressiva com fechamento temporário da parede abdominal deve ser considerada, porém ainda há dúvidas sobre a contribuição dessa técnica. ${ }^{5}$

No que se refere ao transplante de fígado, há fatores relevantes relacionados ao procedimento e aos receptores, para instalação desse quadro no pósoperatório imediato. ${ }^{6}$ Este trabalho revisa a literatura de modo integrado sobre as perspectivas da HIA e da SCA no pós- transplante hepático acerca da prevalência, fatores de risco, monitoramento, complicações e condutas.

\section{MÉTODOS}

Foi realizada busca sistematizada na MEDLINE (via PubMed), Scielo, LILACS (via BVS) e Cochrane, em 24 de julho de 2019. Os termos utilizados foram "liver transplantation", "intra-abdominal hypertension", "abdominal compartment syndrome" incluindo sinônimos, abreviações e adaptações entre os bancos de dados (Figura 1).

Figura 1: Estratégia de busca sistematizada

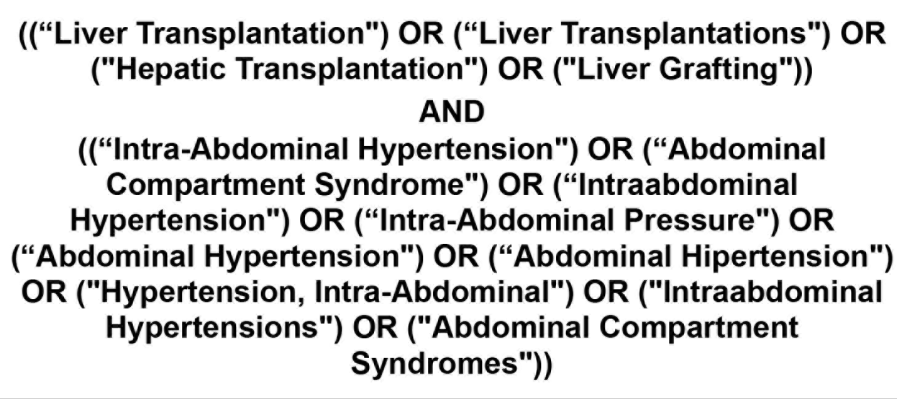

A busca resultou no registro de 84 trabalhos, analisados a partir de uma triagem do título e resumo, e posteriormente, o texto foi submetido aos critérios de elegibilidade. Foram incluídos trabalhos em língua inglesa e portuguesa. Artigos cujo rastreio mostrou duplicidade, procedimento diferente de transplante hepático, transplante hepático combinado com outros órgãos abdominais, sem correlação com PIA, paciente recém-nascido, síndrome compartimental hepática e experimentos em animais foram excluídos. Foram incluídas inicialmente revisões da literatura e as referências dessas publicações foram avaliadas.

Os artigos originais cujos dados apresentaram prevalência, fatores de risco, monitoramento, complicações e manejo da HIA e da SCA durante o pós-operatório do transplante hepático foram incluídos (Figura 2).

Figura 2: Fluxograma da seleção bibliográfica da revisão sistematizada

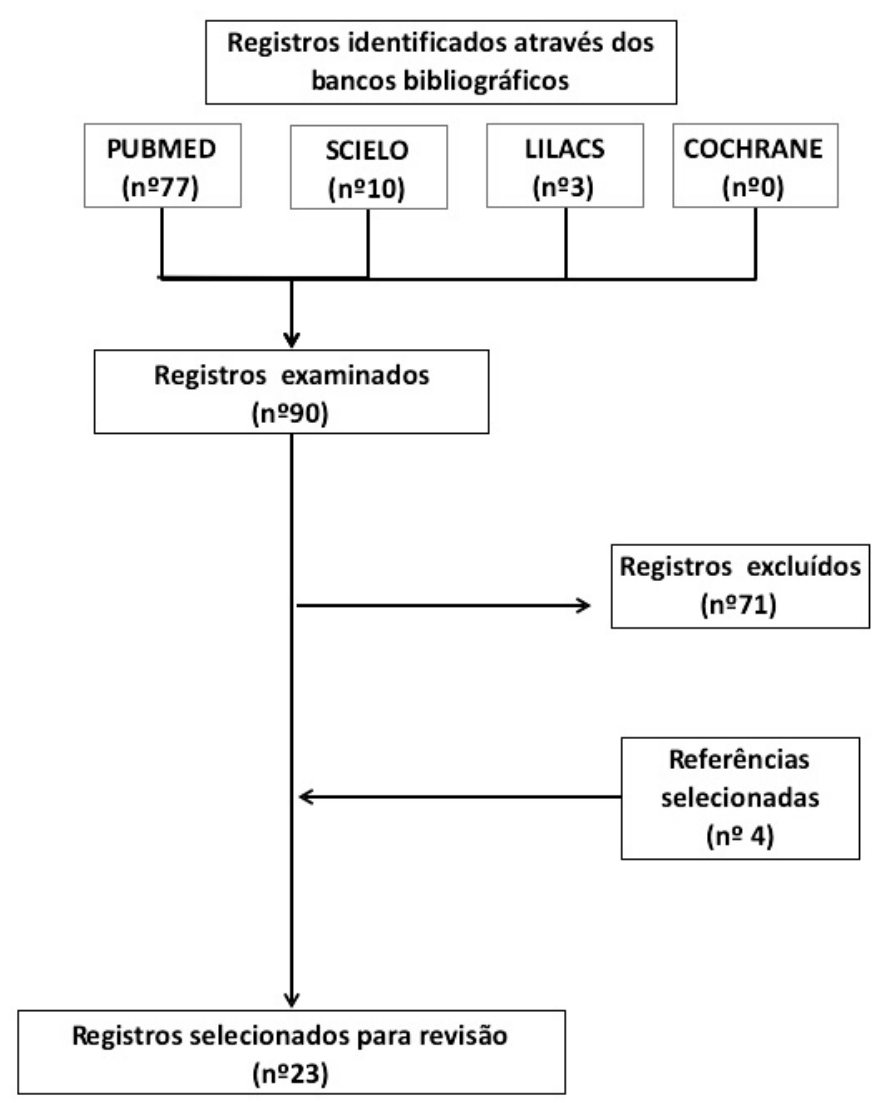

\section{DISCUSSÃO}

\section{Prevalência}

Dentre os estudos selecionados, os artigos originais com dados quantitativos sobre PIA com casuística maior que 20 foram analisados para compor a prevalência da HIA e SCA no pós-transplante hepático. 
Os pacientes apresentaram PIA pré-cirúrgica menor que $6 \mathrm{mmHg}$. Contudo, em um intervalo de 0-72h póstransplante, $38,7 \%$ desenvolveram HIA $(79,2 \%$ nas primeiras 24 horas e $20,8 \%$ entre $24-48$ horas após o transplante). No terceiro dia pós-operatório, os pacientes tiveram regressão do quadro. ${ }^{7}$

Em outro estudo prospectivo com 108 pacientes, os receptores que desenvolveram HIA grau IV tiveram uma redução média de $10 \mathrm{mmHg}$ na PIA até 48-72h após o transplante (de $33,5 \mathrm{mmHg}$ a $23,5 \mathrm{mmHg}$ ). ${ }^{8}$ Deindl et al estudaram retrospectivamente a HIA em pacientes pediátricos submetidos a transplante hepático (nessa população postula-se um valor de corte de $10 \mathrm{mmHg}$ para HIA de acordo com a WSACS). ${ }^{9}$

A revisão dos dados expõe que HIA é uma complicação precoce $(0-72 \mathrm{~h})$ do transplante hepático com prevalência elevada, que varia de acordo com os critérios adotados pelo centro (Tabela 1).

Tabela 1 - Prevalência da Hipertensão Intra-Abdominal e da Síndrome Compartimental Abdominal no transplante hepático

\begin{tabular}{|c|c|c|c|c|c|c|}
\hline Artigo & Ano & $\mathbf{N}$ & Paciente & HIA(\%) & Critério HIA & SCA(\%) \\
\hline Shu et al $^{7}$ & 2007 & 63 & Adulto & $38,70 \%$ & $>20$ mmHg & - \\
\hline Biancofiori et $\mathrm{al}^{8}$ & 2003 & 108 & Adulto & $79 \%$ & $>18 \mathrm{mmHg}$ & $31 \%$ \\
\hline Deindl et $\mathrm{al}^{9}$ & 2019 & 23 & Pediátrico & $87 \%$ & $>10 \mathrm{mmHg}$ & $4,3 \%$ \\
\hline
\end{tabular}

$H I A=$ Hipertensão Intra-Abdominal $; \quad S C A=$ Síndrome Compartimental Abdominal

\section{Fatores de Risco}

Os fatores de risco para o desenvolvimento da HIA observados nos trabalhos em pacientes submetidos a transplante hepático foram explorados nesta sessão. (Fig.3)

Figura 3: Fatores de risco para o desenvolvimento de Hipertensão Intra-Abdominal e da Síndrome Compartimental Abdominal no transplante hepático

\section{FATORES DE RISCO}

Tamanho desproporcional do enxerto

Edema intestinal

Edema do enxerto

Ileo paralítico

Ascite

Sangramento intra-abdominal

Ressuscitação volêmica

O tamanho desproporcional do fígado doado foi associado como fator de risco: Tração das estruturas abdominais, diminuição da complacência da parede abdominal e aumento do volume do conteúdo abdominal podem justificar essa correlação.9-11

O edema intestinal (associado ao clampeamento do sistema porta durante a fase anepática do procedimento e a reposição de líquido operatória) pode agravar a HIA.11,12 O edema do enxerto pós-reperfusão pode causar impossibilidade de fechamento primário da fáscia abdominal. ${ }^{13} \mathrm{O}$ edema visceral subjacente ao transplante hepático atua, portanto, como fator de risco para HIA.

O íleo paralítico pode ser uma causa de aumento do conteúdo abdominal devido à distensão gastrointestinal, e assim deve ser considerado também no desenvolvimento da HIA devido à sua incidência na cirurgia abdominal. ${ }^{14}$
O sangramento e a formação de hematoma na cavidade abdominal são bastante frequentes após o transplante de fígado, devido às características do procedimento e do paciente, e pode levar a um quadro de HIA. Vários relatos reportaram SCA com repercussões clínicas graves, devido ao sangramento intra-abdominal, porém estudos maiores não exploraram especificamente as consequências do sangramento pós-operatório sobre a PIA. ${ }^{15-19}$

A ascite e a reposição maciça de volume intravascular são fatores absolutamente reconhecidos e que podem contribuir para o desenvolvimento da HIA no póstransplante hepático. ${ }^{1}$

$\mathrm{Em}$ relato isolado, a formação de hematoma retroperitoneal provocou SCA $(40 \mathrm{mmHg})$ em virtude de uma colangiopancreatografia retrógrada endoscópica para correção de uma complicação biliar. No trabalho, foi ressaltada a importância do monitoramento do dreno abdominal e da mensuração da PIA. ${ }^{18}$

\section{Monitoramento}

A avaliação da PIA com base em parâmetros antropométricos e clínicos não é confiável. Medidas objetivas são recomendadas a todos os pacientes críticos com fator de risco para desenvolvimento da $\mathrm{HIA} .{ }^{20} \mathrm{O}$ método estabelecido para o monitoramento da PIA é dado de modo indireto, através do cateter vesical.1 Diversos artigos ressaltaram a importância da medida quantitativa da PIA no diagnóstico precoce da SCA e da HIA nos pacientes submetidos ao transplante..$^{7,9,15,18,21}$ Em um dos trabalhos, o tempo médio investido pela equipe médica na mensuração da PIA foi de cerca de dois minutos. ${ }^{9}$ 
Vinte receptores tiveram a PIA avaliada simultaneamente por três vias; sendo duas delas diretas (abdômen superior e inferior) e uma via cateter vesical, com um total de 169 medidas simultâneas. O estudo reafirmou a excelente relação da pressão intravesical com a PIA no compartimento inferior abdominal. Entretanto, destacou que houve certa disparidade com relação ao compartimento abdominal superior. Os autores atribuíram tal diferença ao caráter heterogêneo do conteúdo abdominal (não sendo possível, portanto aplicar a lei de pascal, princípio que afirma ser equivalente à distribuição de pressão em todos os pontos de fluido homogêneo) e que tal diferença pode ser afetada pela posição corporal. ${ }^{22}$ Não há consenso sobre a relevância clínica desse achado (subnotificação da HIA e subdiagnóstico de complicações relevantes), porém ressalta-se a importância da sistematização do método de mensuração quanto à posição do corpo.

Além da medida da pressão, outros métodos foram estudados para avaliar a SCA. A saturação de oxigênio regional de segmentos do corpo foi avaliada como parâmetro no transplante hepático: a dessaturação isolada do membro inferior direito foi significativamente associada ao aumento da PIA. ${ }^{17}$ Esse método de avaliação foi validado também em outras patologias (pancreatite grave). ${ }^{23}$

O corante indocianina verde, cuja depuração é utilizada para avaliação da perfusão hepatoesplênica e da função hepatocelular foi associado com PIA. ${ }^{24,25}$ É sugestivo que a correlação entre o aumento da PIA e o dano hepático associado possa ser melhor avaliada através desse método.

\section{Complicações}

As alterações fisiopatológicas da HIA afetam direta e indiretamente diversos sistemas do corpo, sendo reportadas principalmente alterações no sistema cardiovascular, respiratório, renal, hepatoesplênico e nervoso central. ${ }^{26}$ A resposta clínica dos pacientes submetidos a descompressão abdominal após SCA se assemelha a um quadro de lesão por isquemiareperfusão, conferindo desse modo um mecanismo de má perfusão orgânica à HIA.$^{5}$ Devido às repercussões sistêmicas inerentes a todo paciente acometido de HIA, esta revisão ateve-se às complicações íntimas ao transplante hepático: insuficiência renal aguda e disfunção primária do enxerto.

A insuficiência renal aguda é complicação frequente no pós-transplante hepático, observando-se incidência de $10-25 \%$ e determinando um importante fator de mortalidade. ${ }^{27}$ Diante dos diversos mecanismos sugeridos, a hipovolemia pode ter papel importante nesse cenário. Essa tese foi afastada, em parte, devido à descorrelação com os parâmetros hemodinâmicos que a sustentasse, e ainda à ausência de responsividade da função renal à reposição volêmica nesse cenário.7,11

A HIA cresceu na literatura médica devido aos mecanismos atrelados ao aumento da pressão intraglomerular, ao desvio de sangue do córtex para a medula renal, à elevação da pressão venosa renal e à modulação das vias do balanço hídrico. ${ }^{28}$ Biancofiore et al, em 2003, observaram que pacientes sem alteração na função renal apresentavam PIA média de $19 \mathrm{mmHg}$, enquanto que nos pacientes que desenvolveram insuficiência renal, esse parâmetro aumentou para 27 mmHg. ${ }^{6}$ Em Shu et al, 2007, 45,8\% dos receptores com HIA desenvolveram disfunção renal em contrapartida à $7,9 \%$ dos pacientes que não tiveram aumento da PIA. ${ }^{7}$ Além de outras condições que envolvem o procedimento, de fato, a HIA é fator independente para disfunção renal precoce no pós-transplante hepático.

A disfunção primária do enxerto é complicação também, relevante no transplante hepático, e foi teoricamente associada à HIA. Entretanto. não houve diferença significativa na sobrevida do enxerto, avaliada no sétimo dia pós-operatório no grupo que desenvolveu HIA., Perkins et al 2006, em um estudo com 110 pacientes críticos não relacionados a transplante concluíram não haver associação direta entre a função hepática e aumento da PIA, porém, houve correlação entre o grau de HIA e o de hiperbilirrubinemia. ${ }^{29} \mathrm{O}$ estudo limitou-se a pacientes que não tiveram grandes aumentos da PIA.

Entretanto, a utilização de métodos mais sensíveis para avaliar a perfusão hepatoesplênica, como o tempo de depuração da indocianina verde, foi possível associar ao aumento da PIA com a diminuição da perfusão hepática. ${ }^{24}$ Uma diminuição relevante do fluxo sanguíneo dessa circulação mostrou-se importante durante HIA de baixo e alto grau..$^{15,25}$

A desproporção do enxerto contribui como fator de risco para SCA nos transplantes pediátricos, com crianças pesando menos de $10 \mathrm{~kg}$. Além disso, oferece ao enxerto condições vasculares adversas. ${ }^{10}$ Nesse contexto, portanto, observa-se disfunção hepática secundária à HIA. ${ }^{9,10}$

A HIA causa alteração no fluxo sanguíneo hepático e, subsequentemente, pode haver agravo das comorbidades já existentes a esse órgão, porém não foi possível observar que a HIA pode isoladamente diminuir a sobrevida do enxerto e promover disfunção primária.

\section{Manejo e intervenções}

O manejo clínico da HIA e SCA soma benefícios e preserva contra desfechos desfavoráveis. ${ }^{30}$ As medidas clínicas e procedimentos não cirúrgicos podem prevenir 
a necessidade da laparotomia descompressiva e manutenção do abdome aberto no paciente crítico, enquanto essa abordagem é vital durante SCA.
Condutas de diversas ordens foram estudadas no paciente submetido ao transplante hepático e foram sintetizadas na Tabela 3.

Tabela 2: Intervenções relatadas na literatura para o tratamento da Hipertensão Intra-Abdominal e Síndrome Compartimental após o transplante hepático.

\begin{tabular}{|c|c|c|c|c|}
\hline Artigo & Ano & $\mathbf{N}$ & Pacientes & Intervenção \\
\hline Deindl et $\mathrm{al}^{9}$ & 2019 & 18 & Pediátrico & FT: Proteção sintética \\
\hline Hobeika et al ${ }^{31}$ & 2017 & 46 & Adulto & FT: VAC vs FAE \\
\hline Assirati et al $^{32}$ & 2016 & 11 & Adulto & FT: VAC \\
\hline Akdur et $\mathrm{al}^{10}$ & 2015 & 17 & Pediátrico & FT: Bolsa Bogotá e FAE \\
\hline Karpelowsky et al $\left.\right|^{33}$ & 2009 & 10 & Pediátrico & FD: Membrana biológica \\
\hline Singh et $\mathrm{al}^{13}$ & 2008 & 10 & Adulto & FD: Matriz de pele \\
\hline Petersen et $\mathrm{al}^{14}$ & 2019 & 3 & Pediátrico & Neostigmina \\
\hline Nagy et $\mathrm{al}^{16}$ & 2019 & 1 & Adulto & LD + Hepatectomia parcial \\
\hline Zhang et $\mathrm{al}^{21}$ & 2009 & 1 & Adulto & LD + Colectomia parcial \\
\hline Milanchi et al ${ }^{18}$ & 2007 & 1 & Adulto & LD \\
\hline Handschin et $\mathrm{al}^{15}$ & 2005 & 1 & Adulto & $L D+V A C$ \\
\hline Shelly et al12 & 1987 & 3 & Adulto & LD \\
\hline
\end{tabular}

FT: fechamento temporário; VAC: vacuum-assisted closure; FAE: fechamento apenas da epiderme;

FD: fechamento definitivo primário; LD: laparotomia descompressiva

O fechamento primário da fáscia abdominal, em alguns casos após o transplante hepático, é impossibilitado pelo aumento do conteúdo abdominal (desproporção do enxerto, edema intestinal e hepático). Trata-se de uma condição infrequente, sendo reportada uma prevalência de 2,9\%-3,1\%. . $^{31,32}$ Várias técnicas têm sido usadas com objetivo de aumentar a complacência da parede abdominal nessas circunstâncias (Tabela 2).

A manutenção do abdome aberto é factível e posterga o fechamento da parede abdominal durante a reabsorção do edema visceral, entretanto, o fechamento definitivo deve ser realizado precocemente. ${ }^{31} \mathrm{O}$ desenvolvimento da técnica de terapia de pressão negativa (VAC) tornou-a indispensável no tratamento de feridas complexas, e o método comprovou-se superior também no transplante hepático quanto ao tempo necessário de manutenção do abdômen aberto. ${ }^{31,32}$

O fechamento temporário da parede abdominal, com a utilização de material sintético até o fechamento definitivo, pode ser realizado com Vicryl, politetrafluoretileno expandido, silício e outros. No pós-operatório imediato do transplante pediátrico, não foi observada diferença significativa na PIA, assim como na pressão de perfusão abdominal, entre fechamento primário e temporário, e foi observado um aumento discreto na PIA, após cada redução escalonada da área aberta. Deindl et al propuseram que a PIA pode ter um papel importante como guia para a redução e fechamento definitivo daqueles pacientes com impossibilidade de fechamento primário no transplante hepático. ${ }^{9}$

O fechamento temporário da parede abdominal, através do fechamento apenas da epiderme ou com o uso da bolsa de Bogotá, comprovou segurança e eficácia inicial; as técnicas foram utilizadas para prevenir a SCA em pacientes pediátricos $(<10 \mathrm{~kg})$ submetidos ao transplante hepático com enxertos desproporcionais (razão de massa maior que $4 \%$ do peso do receptor). ${ }^{10}$

Nesse estudo, a impossibilidade do fechamento primário, o desenvolvimento HIA e a ultrassonografia com doppler (antes e imediatamente após do fechamento da fáscia abdominal) foram usados como parâmetro para indicar a manutenção do abdome aberto. A principal complicação da técnica foi o desenvolvimento de hérnia abdominal, mas não afetou a sobrevivência do enxerto e nem do receptor. Foi sugerido que esse método pode ser uma alternativa à redução em monossegmentos no transplante com doadores vivos. ${ }^{10}$ 
Em Zhang et al, 2009, um relato de caso de uma paciente transplantada, portadora de dolicocólon, foi submetida ao abdome aberto, após o diagnóstico de SCA e, após cinco dias, ao fechamento definitivo subsequente à colectomia parcial. ${ }^{21}$

Os métodos acompanhados da síntese primária definitiva que aumentam a complacência da parede abdominal são, também, uma alternativa que pode prevenir a SCA e as complicações inerentes do abdômen aberto das técnicas anteriores.

Foi estudado no pós-transplante hepático o uso de materiais derivados de pele humana e de membrana da submucosa suína como substituto da fáscia abdominal com resultados iniciais favoráveis. ${ }^{13,33}$ Ambas as técnicas previnem o receptor de uma nova abordagem cirúrgica e, teoricamente, podem diminuir a incidência de hérnia incisional. A utilização do método varia consideravelmente com a acessibilidade e a experiência do centro, e não há evidência sobre o uso multicêntrico dessas técnicas.

A HIA associada ao íleo paralítico no transplante hepático foi tratada com o uso de Neostigmina em três casos de receptores pediátricos. ${ }^{14} \mathrm{Um}$ dos pacientes evoluiu para SCA e foi submetido à laparotomia descompressiva e fechamento temporário da parede abdominal. Os pacientes toleraram a infusão sem efeitos adversos e tiveram boa resolução da distensão abdominal. Os resultados fazem parte da transferência do uso de Neostigmina já indicado para o tratamento da HIA, conforme a WSACS recomenda ao transplante de fígado. ${ }^{1}$
O resultado da laparotomia descompressiva nos pacientes que desenvolveram SCA após o transplante hepático foi a redução imediata da PIA. ${ }^{9,12,15,16,18,21} \mathrm{O}$ sangramento maciço intra-abdominal foi reportado em quase todos os trabalhos. ${ }^{12,16,18,21} \mathrm{O}$ alívio abrupto da PIA demonstra reperfusão da circulação hepatoesplênica, diminuição da resistência vascular periférica e hipotensão. ${ }^{12}$ Nos diversos cenários clínicos da SCA, em revisão sistematizada Van Damme e De Waele concluíram que a laparotomia descompressiva tem um papel importante na redução da PIA, na função renal e no controle hemodinâmico/respiratório; entretanto, não se sabe exatamente quais os pacientes que podem beneficiar-se mais da laparotomia descompressiva, porque os resultados entre os estudos são bastante variados e a mortalidade remanescente ainda é alta. ${ }^{5}$

\section{CONCLUSÃO}

A HIA e a SCA são complicações frequentes que podem determinar aumento da morbimortalidade no pós-transplante hepático. Há diversos fatores de risco inerentes com procedimento para o desenvolvimento dessas condições e devem, portanto, ser ativamente monitoradas através da PIA medida pelo cateter vesical. A HIA é fator independente para insuficiência renal aguda e pode provocar prejuízo à perfusão hepática. Medidas para o controle e a prevenção da HIA e da SCA devem ser tomadas no pós-operatório imediato, incluindo métodos clínicos e cirúrgicos. A manutenção do abdome aberto e a laparotomia descompressiva destacam-se nesse cenário.

\section{ABSTRACT}

Introduction:In the postoperative period of liver transplantation there may be an increase in volume contained in the abdomen, massive fluid replacement, intraperitoneal blood accumulation and gastrointestinal distension: these conditions are relevant for Intra-Abdominal Hypertension (HIA) and Abdominal Compartment Syndrome (SCA) development. Evidence on this topic is in progress. The aim of this study was to provide an integrative view of the literature on the prospects for HIA and SCA in liver transplantation. Methods: A systematic search was carried out on MEDLINE (via PubMed), Scielo, LILACS (via BVS) and Cochrane on July 24, 2019. The works were selected by inclusion and exclusion criteria proposed according to the purpose of this review, and were divided in five categories: prevalence, risk factors, monitoring, complications and management. Discussion: HIA and ACS are frequent complications, which can determine an increase in morbidity and mortality in post-liver transplantation. There are several risk factors inherent to the procedure to the development of these conditions, and therefore, it should be actively monitored through the IAP measured by a bladder catheter. HIA is an independent factor for acute renal failure, and it can contribute to the impairing of liver perfusion. Measures for the HIA and ACS control and prevention should be taken in the immediate postoperative period, including both clinical and surgical methods. Maintenance of open abdomen and decompressive laparotomy stand out in this scenario.

Keywords: Liver Transplantation; Intra-abdominal Hypertension; Abdominal Compartment Syndrome. 


\section{REFERÊNCIAS}

1. Kirkpatrick AW, Roberts DJ, De Waele J, Jaeschke R, Malbrain MLNG, De Keulenaer B, et al. Intra-abdominal hypertension and the abdominal compartment syndrome: updated consensus definitions and clinical practice guidelines from the World Society of the Abdominal Compartment Syndrome. Intensive Care Med. 2013;39(7):1190-206.

2. Malbrain ML, De Waele J, De Keulenaer BL. What every ICU clinician needs to know about the cardiovascular effects caused by abdominal hypertension. Anaesthesiol Inten Ther. 2015;47(4):388-99.

3. Malbrain MLNG, Chiumello D, Pelosi P, Bihari D, Innes R, Ranieri VM, et al. Incidence and prognosis of intraabdominal hypertension in a mixed population of critically ill patients: a multiple-center epidemiological study. Crit Care Med. 2005;33(2):315-22.

4. Kyoung K-H, Hong S-K. The duration of intra-abdominal hypertension strongly predicts outcomes for the critically ill surgical patients: a prospective observational study. World J Emerg Surg WJES. 2015;10:22.

5. Van Damme L, De Waele JJ. Effect of decompressive laparotomy on organ function in patients with abdominal compartment syndrome: a systematic review and metaanalysis. Crit Care [Internet]. 25 de julho de 2018 [citado 23 de junho de 2020];22. Disponível em: https://www.ncbi.nlm. nih.gov/pmc/articles/PMC6060511/

6. Biancofiore G, Bindi ML, Romanelli AM, Boldrini A, Consani $\mathrm{G}$, Bisà $\mathrm{M}$, et al. Intra-abdominal pressure monitoring in liver transplant recipients: a prospective study. Intensive Care Med. 2003;29(1):30-6.

7. Shu M, Peng C, Chen H, Shen B, Zhou G, Shen C, et al. Intra-abdominal hypertension is an independent cause of acute renal failure after orthotopic liver transplantation. Front Med China. 2007;1(2):167-72.

8. Biancofiore G, Bindi L, Romanelli AM, Bisà M, Boldrini A, Consani $G$, et al. Renal failure and abdominal hypertension after liver transplantation: determination of critical intraabdominal pressure. Liver Transplant Off Publ Am Assoc Study Liver Dis Int Liver Transplant Soc. 2002;8(12):117581.

9. Deindl P, Wagner J, Herden U, Schulz $\square$ Jürgensen S, Schild R, Vettorazzi E, et al. Monitoring intra-abdominal pressure after liver transplantation in children. Pediatr Transplant. 2019;23(7):e13565.

10. Akdur A, Kirnap M, Ozcay F, Sezgin A, Ayvazoglu Soy HE, Karakayali Yarbug F, et al. Large-for-size liver transplant: a single-center experience. Exp Clin Transplant Off J Middle East Soc Organ Transplant. 2015;13 Suppl 1:108-10.

11. Biancofiore $G$, Bindi ML, Romanelli AM, Bisà M, Boldrini A, Consani G, et al. Postoperative intra-abdominal pressure and renal function after liver transplantation. Arch Surg Chic III 1960. 2003;138(7):703-6.
12. Shelly MP, Robinson AA, Hesford JW, Park GR. Haemodynamic effects following surgical release of increased intra-abdominal pressure. $\mathrm{Br} \mathrm{J}$ Anaesth. 1987;59(6):800-5.

13. Singh MK, Rocca JP, Rochon C, Facciuto ME, Sheiner PA, Rodriguez-Davalos MI. Open abdomen management with human acellular dermal matrix in liver transplant recipients. Transplant Proc. 2008;40(10):3541-4.

14. Petersen PC, Balakrishnan B, Vitola B, Hong JC. Case report series of a novel application of neostigmine to successfully relieve refractory ileus status post-pediatric orthotopic liver transplantation. Pediatr Transplant. 2019;23(7):e13564.

15. Handschin AE, Weber M, Renner E, Clavien P-A. Abdominal compartment syndrome after liver transplantation. Liver Transplant Off Publ Am Assoc Study Liver Dis Int Liver Transplant Soc. 2005;11(1):98100.

16. Nagy G, Görög D, Kóbori L, Mihály E, Piros L, Pőcze B, et al. Abdominal Compartment Syndrome After Liver Transplant in Drug-Induced Acute Liver Failure: A Case Report. Transplant Proc. 2019;51(4):1289-92.

17. Hu T, Collin Y, Lapointe R, Carrier FM, Massicotte L, Fortier A, et al. Preliminary Experience in Combined Somatic and Cerebral Oximetry Monitoring in Liver Transplantation. J Cardiothorac Vasc Anesth. 2018;32(1):73-84.

18. Milanchi S, Magner D, Lo SK, Klein AS, Colquhoun $\mathrm{SD}$, Nissen NN. Abdominal compartment syndrome secondary to retroperitoneal hematoma as a complication of ERCP after liver transplantation. Transplant Proc. 2007;39(1):169-71.

19. Chan-Dominy AC, Rahiman SN, Anders M, Butt W. Extracorporeal membrane oxygenation and severe portopulmonary hypertension following liver transplantation: brief report. Int J Artif Organs. 2015;38(6):337-42.

20. Sugrue M, Bauman A, Jones F, Bishop G, Flabouris A, Parr M, et al. Clinical examination is an inaccurate predictor of intraabdominal pressure. World J Surg. dezembro de 2002;26(12):1428-31.

21. Zhang W, Wang K, Qian X, Xia Y, Zheng C, Zuo X, et al. Abdominal compartment syndrome associated with capillary leak syndrome after liver transplantation. Transplant Proc. 2009;41(9):3927-30.

22. Cresswell $A B$, Jassem $W$, Srinivasan $P$, Prachalias $A A$, Sizer E, Burnal W, et al. The effect of body position on compartmental intra-abdominal pressure following liver transplantation. Ann Intensive Care. 2012;2 Suppl 1:S12.

23. Lecluyse V, Couture EJ, Denault AY. A Proposed Approach to Cerebral and Somatic Desaturation in the Intensive Care Unit: Preliminary Experience and Review. J Cardiothorac Vasc Anesth. 2017;31(5):1805-9. 
24. Malbrain ML, Viaene D, Kortgen A, De Laet I, Dits $H$, Van Regenmortel N, et al. Relationship between intraabdominal pressure and indocyanine green plasma disappearance rate: hepatic perfusion may be impaired in critically ill patients with intra-abdominal hypertension. Ann Intensive Care. 2012;2 Suppl 1:S19.

25. Seibel A, Sakka SG. [Indocyanine green plasma disappearance rate: estimation of abdominal perfusion disturbances]. Anaesthesist. 2010;59(12):1091-8.

26. Malbrain MLNG. Is it wise not to think about intraabdominal hypertension in the ICU? Curr Opin Crit Care. 2004;10(2):132-45.

27. Gainza FJ, Valdivieso A, Quintanilla N, Errazti G, Gastaca $\mathrm{M}$, Campo M, et al. Evaluation of acute renal failure in the liver transplantation perioperative period: incidence and impact. Transplant Proc. 2002;34(1):250-1.

28. Doty JM, Saggi BH, Blocher CR, Fakhry I, Gehr T, Sica D, et al. Effects of increased renal parenchymal pressure on renal function. J Trauma. 2000;48(5):874-7.
29. Perkins JD. Abdominal compartment syndrome: is the liver involved? Liver Transplant Off Publ Am Assoc Study Liver Dis Int Liver Transplant Soc. 2006;12(10):1555-6.

30. Cheatham ML. Nonoperative management of intraabdominal hypertension and abdominal compartment syndrome. World J Surg. 2009;33(6):1116-22.

31. Hobeika C, Allard M-A, Bucur P-O, Naili S, Sa Cunha A, Cherqui D, et al. Management of the Open Abdomen after Liver Transplantation. World J Surg. 2017;41(12):3199_ 204.

32. Assirati G, Serra V, Tarantino G, Aldrovandi S, Ballarin $R$, Magistri P, et al. Vacuum-Assisted Closure Therapy in Patients Undergoing Liver Transplantation With Necessity to Maintain Open Abdomen. Transplant Proc. 2016;48(2):383-5.

33. Karpelowsky JS, Thomas G, Shun A. Definitive abdominal wall closure using a porcine intestinal submucosa biodegradable membrane in pediatric transplantation. Pediatr Transplant. 2009;13(3):285-9. 